\title{
Fast high-fidelity geometric quantum control with quantum brachistochrones
}

\author{
Yang Dong, Ce Feng, Yu Zheng, Xiang-Dong Chen, Guang-Can Guo, and Fang-Wen Sun ${ }^{*}$ \\ CAS Key Laboratory of Quantum Information, University of Science and Technology of China, Hefei 230026, People's Republic of China \\ and CAS Center for Excellence in Quantum Information and Quantum Physics, University of Science and Technology of China, \\ Hefei 230026, People's Republic of China
}

(Received 8 April 2021; accepted 1 December 2021; published 13 December 2021)

\begin{abstract}
We experimentally demonstrate fast and high-fidelity geometric control of a quantum system with the brachistochrone method on hybrid spin registers in diamond. Based on the time-optimal universal geometric control, single geometric gates with fidelities over $99.2 \%$ on the spin state of the nitrogen-vacancy center are realized with average durations shortened by $21.5 \%$ compared with the conventional geometric method. The fidelity of the fast geometric two-qubit gate exceeds $96.5 \%$ on the hybrid spin registers. With the fast and high-fidelity universal set of geometric gates available, we implement a quantum entanglement-enhanced phase estimation algorithm and demonstrate the Heisenberg quantum limit of phase estimation at room temperature. Hence our results show that high-fidelity quantum control based on a fast geometric route will be a versatile tool for broad applications of quantum information processing in practice.
\end{abstract}

DOI: 10.1103/PhysRevResearch.3.043177

\section{INTRODUCTION}

Quantum information processing (QIP), which can provide an unprecedented supremacy $[1,2]$ over its classical counterpart in searching algorithms [3,4], simulations [5,6], metrology [7-12], and secure communication [13], relies seriously on high-quality quantum operations [14-17]. Meanwhile, the geometric phase [18] is currently a central topic not only in quantum physics but also in various scientific fields including optics, electronics, nanotechnology, chemical reactions, and materials science. With the built-in noise-resilience feature, the geometric-phase-based holonomic quantum gate is believed to be an ideal way to build a fault-tolerant universal quantum computer [19,20]. Also, geometric phase can be used in the quantum sensing of the magnetic field with high sensitivity and large dynamic range [21]. Based on adiabatic or nonadiabatic cyclical paths, it has been proposed as a promising method to realize high-fidelity and robust manipulation in theory [20,22-26], along with experimental demonstrations in various physical platforms, including superconducting circuits [27-29], nuclear magnetic resonance [30,31], and solid-state defects in diamond [32-36].

However, the duration times of these geometric operations are still much longer than those of dynamical cases, especially for the adiabatic geometric quantum computation (GQC) protocol, resulting in more decoherence degrading

\footnotetext{
*fwsun@ustc.edu.cn

Published by the American Physical Society under the terms of the Creative Commons Attribution 4.0 International license. Further distribution of this work must maintain attribution to the author(s) and the published article's title, journal citation, and DOI.
}

quantum states over time [25,28,31,37-40]. Besides fighting decoherence [38,41-43], long operation durations will severely reduce the detection sensitivity and bandwidth for quantum metrology based on full GQC. Recently, an experimental demonstration of an optical fast geometric single-qubit gate was reported $[44,45]$. Because the quantum system scalability, closed evolution path, and the optimization of fidelity and time cost should be satisfied simultaneously [46-50], an accurate and fast universal set of geometric gates was not implemented in the experiment. Moreover, it is challenging to improve the performance of quantum metrology with fast GQC in practice.

Here, by employing the quantum brachistochrone equation (QBE) [51-54], we implement fast and high-fidelity geometric quantum gates based on the time-optimal control (TOC) method. In the experiment, we demonstrate a single-loop brachistochrone nonadiabatic holonomic quantum computation (B-NHQC) in a prototype hybrid quantum system: a two-qubit register consisting of a nitrogen-vacancy (NV) electron and a ${ }^{14} \mathrm{~N}$ nuclear spin in diamond. Fast geometric single-qubit gates with fidelities over $99.2 \%$ on electron spin are realized with the average cutoff duration shortened by $21.5 \%$, compared with the conventional NHQC method. The nontrivial two-qubit geometric gate is also realized with a fidelity of $96.5 \%$, which is much faster than dynamical cases. Based on the fast and high-fidelity universal set of geometric gates, we further implement the entanglement-enhanced phase estimation algorithm with an NV center. In addition to the demonstration of the Heisenberg quantum limit (HQL) with the two entangled qubits [7-12], the best detection sensitivity and bandwidth are also improved in the experiment. Hence, our results suggest that B-NHQC-based gates can provide a promising route to achieve fast and precise universal quantum control and make an important step towards practical high-performance application of QIP. 


\section{B-NHQC SCHEME WITH NV CENTER}

In the experiment, we construct the geometric quantum gate [31-33,36] with the NV center in diamond [15,41], a promising candidate for a scalable quantum register. By applying a magnetic field along the axis of the NV center, the electron spin-triplet states form a $V$-type energy level system. We encode $\left|m_{s}=-1\right\rangle \equiv|0\rangle$ and $\left|m_{s}=1\right\rangle \equiv|1\rangle$ as the qubit basis states and use $\left|m_{s}=0\right\rangle \equiv|a\rangle$ as an ancillary state for the geometric manipulation of the qubit. Two microwave (MW) fields with frequencies $\omega_{1(2)}$ and initial phases $\phi_{1(2)}$ are used to couple the sequential transitions $|0\rangle \leftrightarrow|a\rangle(|a\rangle \leftrightarrow|1\rangle)$ with corresponding Rabi frequencies $\Omega_{1(2)}$, as described by

$$
H=\frac{\Omega(t)}{2} e^{i \phi_{2}}|b\rangle\langle a|+\text { H.c. }
$$

where $|b\rangle=e^{i \phi} \sin \frac{\theta}{2}|0\rangle+\cos \frac{\theta}{2}|1\rangle$ is a bright state with $\tan \frac{\theta}{2}=\frac{\Omega_{1}}{\Omega_{2}}$. Here, $\phi=\phi_{1}-\phi_{2}$, and $\Omega(t)=\sqrt{\Omega_{1}^{2}(t)+\Omega_{2}^{2}(t)}$ is the Rabi frequency. Therefore the bright state $|b\rangle$ interacts with the state $|a\rangle$, while it decouples from the dark state $|d\rangle=\cos \frac{\theta}{2}|0\rangle-e^{-i \phi} \sin \frac{\theta}{2}|1\rangle$.

The NHQC scheme $[19,26,36]$ can be realized with a single-loop scenario by engineering the quantum system to evolve along with a red slice-shaped path in the Bloch sphere, as shown in Fig. 1(a). In the qubit computational basis $\{|0\rangle,|1\rangle\}$, the gate operation is $U_{G}\left(\gamma, \theta, \phi_{2}\right)=$ $e^{i(\gamma / 2)} e^{-i(\gamma / 2) \vec{n} \cdot \vec{\sigma}}$, which describes a rotation around the $\vec{n}=$ ( $\sin \theta \cos \phi, \sin \theta \sin \phi, \cos \theta$ ) axis by a $\gamma$ angle. Therefore the duration times are the same for all gates, as shown in Figs. 1(b) and 1(c). However, in realistic physical systems, the ever-present decoherence from the environment degrades the qualities of quantum states and operations over time. Therefore generating the fastest possible cyclic path evolution by TOC becomes a preferable choice for realizing precise quantum controls [48,51-53]. The accurate path with the minimal time cost can be obtained by solving the QBE together with the Schrödinger equation. Here, the QBE can be written as $\dot{F}=-i[H, F]$, where $F=\partial L_{C} / \partial H$ and $L_{C}=$ $\sum_{j} \lambda_{j} f_{j}(H)$ with the Lagrange multiplier $\lambda_{j}$. One physically relevant constraint is the finite operation speed $[55,56]$, which is described as $f_{1}[H(t)] \equiv \frac{1}{2}\left[\operatorname{Tr}\left[H(t)^{2}\right]-\frac{\Omega(t)^{2}}{2}\right]=0$. The other constraint is $f_{2}[H(t)] \equiv \operatorname{Tr}\left[H(t) \sigma_{z}\right]=0$ since we cannot directly implement the independent $\sigma_{z}$ operation in the experiment. A simple minimum-time solution $[57,58]$ to this QBE is $\phi_{2}(t)=2(\gamma-\pi) t / \tau$, with the minimum gate duration time of $\tau_{\min }=2 \sqrt{\pi^{2}-(\pi-\gamma)^{2}} / \Omega$, which increases with the geometric phase, as shown in Figs. 1(b) and 1(c). Under this condition, the single-loop geometric gate $U_{G}$ can be implemented through the green spherical-crown-shaped path as shown in Fig. 1(a), which is less time-consuming than the conventional NHQC scheme as shown in red.

\section{SINGLE-QUBIT GATE}

By setting $\Omega(t)=12.5 \mathrm{MHz}$, we experimentally demonstrate the $R(\hat{z}, \theta)$ operation with $\theta=\pi / 8, \pi / 4$, and $\pi$ based on the B-NHQC. The time cost is less than that with the NHQC scheme, on average, by $21.5 \%$, as shown in Fig. 1(c). We implement the target $T=U_{G}(\pi / 4,0,0)$ gate operation
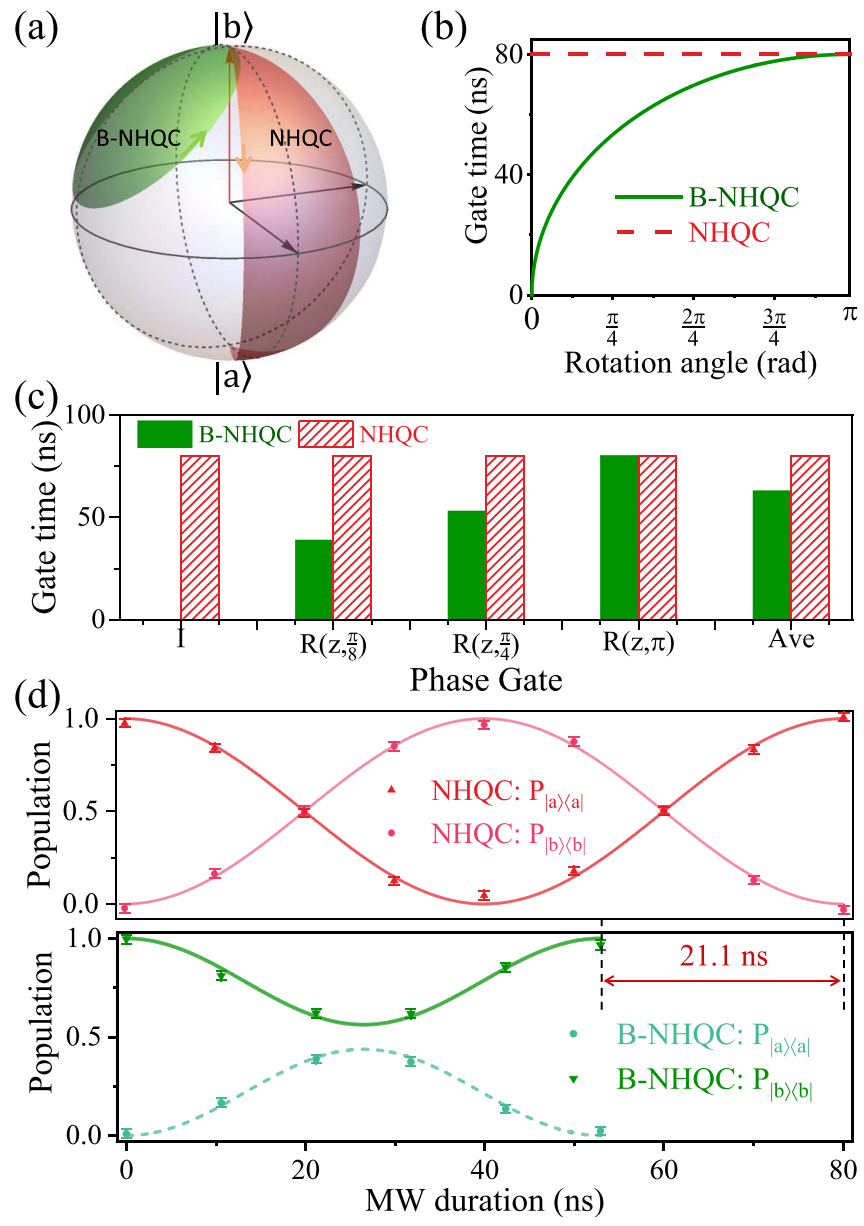

FIG. 1. (a) A geometric picture of the quantum gates in the Bloch sphere representation shows the evolution paths of B-NHQC (green line) and conventional NHQC (red line) schemes. (b) Comparison of time costs for target gate operator $R(\hat{z}, \theta)$ with $\theta \in[0, \pi]$. The Rabi frequency is the same for both protocols. (c) Comparison of the experimental gate time for $\theta=\pi / 8, \pi / 4$, and $\pi$. (d) State evolutions during $T=R\left(\hat{z}, \frac{\pi}{4}\right)$ with B-NHQC and the conventional NHQC paths. The initial state is $|b\rangle=(|0\rangle+|1\rangle) / \sqrt{2}$. The time of the geometric gate $T$ with the TOC is $21.1 \mathrm{~ns}(26.4 \%)$ shorter than that of the conventional scheme.

with both methods as shown in Fig. 1(d). The duration of the $T$ gate is only $73.6 \%$ of that with the NHQC scheme. Specifically, the short gate duration in the B-NHQC scheme is beneficial to reduce the transverse dephasing process of the NV center.

We characterize the single-qubit B-NHQC gates through a standard quantum process tomography (QPT) method [43] on six specific geometric gates $\{I, X / 2, X, Y / 2, Y, T\}$, with an average fidelity of $0.984(4)$. The major contribution to the QPT infidelity comes from state preparation and detection errors $[32,33,35,59]$. As it is performed through MW-induced dynamical phase shifts, the QPT is more prone to noise, parameter imperfection, and decoherence effects than the process in the B-NHQC gate. Therefore, to characterize the intrinsic B-NHQC gate error, we concatenate a series of gates and analyze the fidelity decay as the number of gates increases only [32,36]. As shown in Fig. 2, we implement $X$ and $Y$ gates 


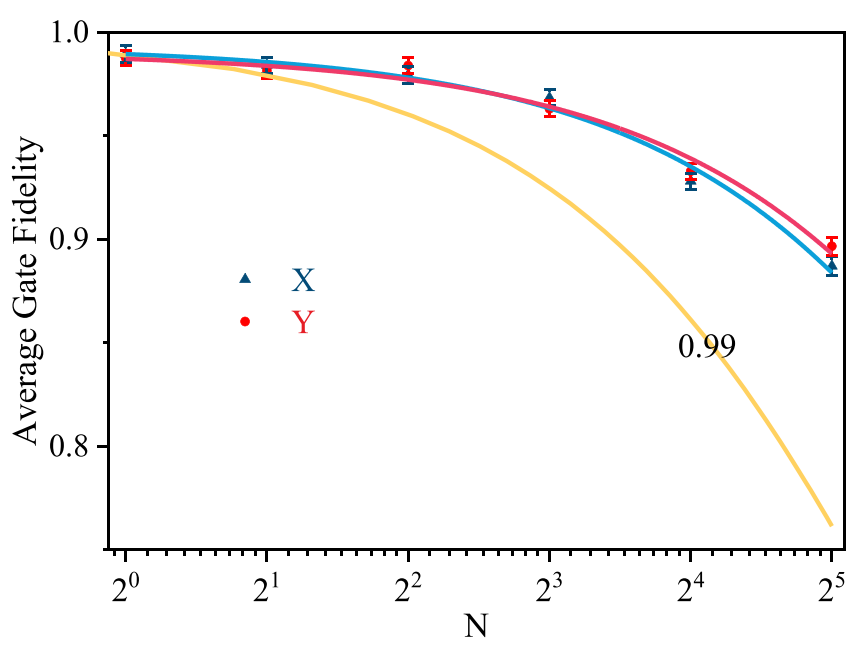

FIG. 2. The decays of the fidelity of the B-NHQC $X$ and $Y$ gates are obtained via QPT and fitted by the solid curves.

successively on the electron spins and perform QPT when the gates are repeated $N$ times. By assuming an exponential model for the state fidelity [14,32,60], the average fidelity of $N$ B-NHQC gates can be calculated as $F_{N}=\left[1+\left(1-\varepsilon_{i f}\right)(1-\right.$ $\left.p)^{N}\right] / 2$, where $\varepsilon_{\text {if }}$ describes errors in the state preparation and measurement and $p$ shows the average error per B-NHQC gate $[32,60]$. We estimate the average fidelity of the $X$ and $Y$ gates to be $F_{X}=1-p_{X}=0.9922(4)$ and $F_{Y}=0.9923(4)$ from the experimental data, which exceed the threshold of the necessary fidelity for the realization of state-of-the-art error correction codes based on surface codes [59].

\section{TWO-QUBIT GATE}

Universal geometric control of qubits always requires a nontrivial two-qubit gate. Here, in the construction of the two-qubit gate, we use the electron spin state as the target state and the nitrogen nuclear spin $\left({ }^{14} \mathrm{~N}\right)$ as the control qubit. For this type of solid hybrid spin system, the two-qubit gate poses some challenges in the experiment, mostly because the characteristic properties of the two types of spins differ by three orders of magnitude. In the experiment, we implement a controlled-rotation gate with MW selective operation, which is equivalent to a controlled-NOT gate [61-63]. Similarly, we encode $\left|m_{I}=-1\right\rangle \equiv|0\rangle$ and $\left|m_{I}=1\right\rangle \equiv|1\rangle$ as the qubit basis states and use $\left|m_{I}=0\right\rangle \equiv|a\rangle$ as an ancillary state for the geometric manipulation of the ${ }^{14} \mathrm{~N}$ nuclear spin. A typical controlled $Y(\mathrm{C}-\mathrm{Y})$ gate can be represented as $U_{\mathrm{C}-\mathrm{Y}}=$ $\left(\begin{array}{cc}\mathbf{1} & \mathbf{0} \\ \mathbf{0} & \mathbf{R}_{\mathbf{y}}(\pi)\end{array}\right)$, where $\mathbf{1}, \mathbf{0}$, and $\mathbf{R}_{\mathbf{y}}(\pi)$ represent $2 \times 2$ matrices corresponding to the unit operator, zero matrix, and a rotation matrix around the $y$ axis with $\mathbf{R}_{\mathbf{y}}(\pi)=e^{-i \pi \sigma_{y} / 2}$, respectively. Hence the electron spin will be rotated if the ${ }^{14} \mathrm{~N}$ nuclear spin state is $\left|m_{I}=1\right\rangle$ as shown in Fig. 3(a).

Since the inhomogeneous broadening effects of the NV center's electron spin degrade the performance of the entangled gate, we implement the geometric $I$ gate if the ${ }^{14} \mathrm{~N}$ nuclear spin state is $\left|m_{I}=-1\right\rangle$ with selective MW field driving instead of doing nothing as shown in Fig. 3(b). In this way, the coherence of this hybrid system can be protected by the dressed states [64]. Using this method, the (a)

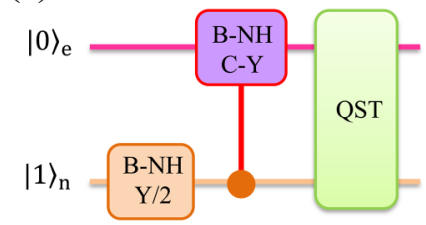

(b)

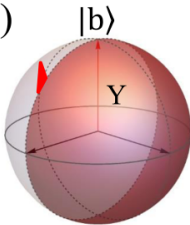

$|0\rangle$ |b〉

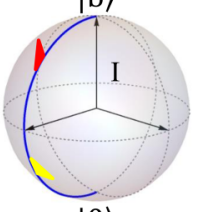

$|0\rangle$
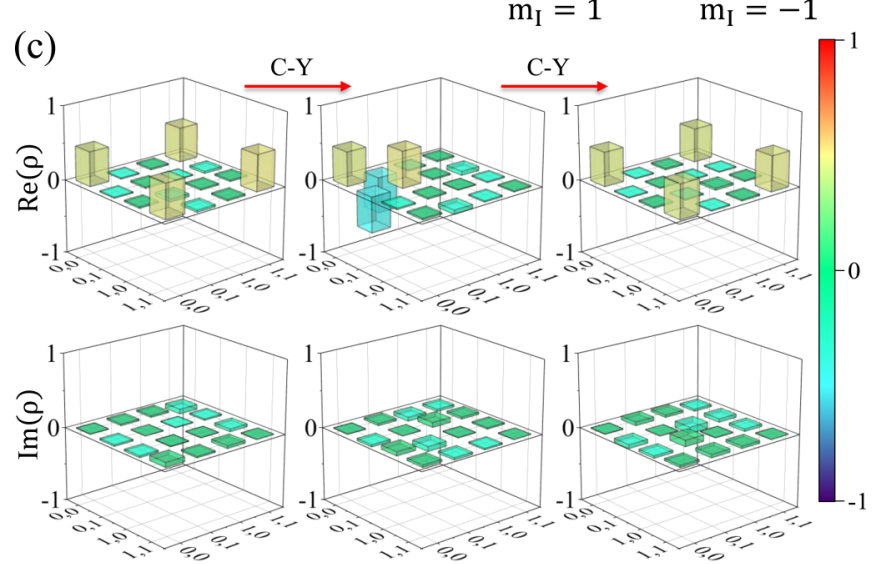

(d)

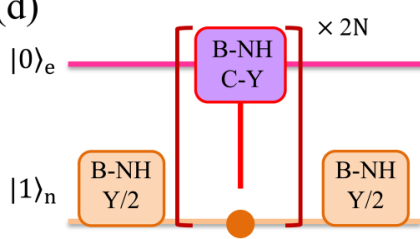

(e)

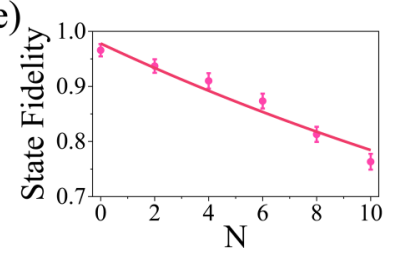

FIG. 3. (a) The quantum circuit to evaluate the B-NHQC twoqubit gate. (b) Dynamical process of the B-NHQC C-Y gate with Bloch sphere. When the ${ }^{14} \mathrm{~N}$ nuclear spin state is at $\left|m_{I}=1\right\rangle$, the geometric $Y$ gate is applied on the electron spin with selective MW. For the geometric $I$ gate on the basis space $\left\{\left|0, m_{I}=-1\right\rangle,\left|b, m_{I}=-1\right\rangle\right\}$ with cyclic evolution time of $\tau, \phi_{2}=0$ during the first time interval $[0, \tau / 2)$, and the corresponding evolution operator is $U_{a}=|d\rangle\langle d|-$ $i(|b\rangle\langle a|+| a\rangle\langle b|)$. For the following part $t \in[\tau / 2, \tau], \phi_{2}=\pi$ and $U_{b}=|d\rangle\langle d|+i(|b\rangle\langle a|+| a\rangle\langle b|)$. In the end, $I=U_{b} U_{a}$ is achieved. (c) The density matrices of the two-qubit states reconstructed by the two-qubit QST to show the transformation by the B-NHQC C-Y gate. The bars indicate the values of the matrix elements. (d) The quantum circuit to apply $N$ B-NHQC C-Y gates (B-NH C-Y). (e) State fidelities after $N$ applications of the C-Y operation. Pink dots are the final state fidelities for even $N$, which are fitted with the red curve.

geometric entangled state between the electron and ${ }^{14} \mathrm{~N}$ nuclear spin is prepared and measured in the Bell state $|\Phi\rangle=$ $(|00\rangle+|11\rangle) / \sqrt{2}$ with a state fidelity of $F_{s}=0.947(8)$ by the quantum state tomography (QST) method. By applying the B-NHQC C-Y gate repeatedly, the state of the hybrid system is coherently transferred between entangled and separable states, as shown in Fig. 3(b). In such a hybrid spin system, coherent operation of the nuclear spin costs much more time than coherent operation of the electron spin. The typical operation time $\left(t_{\pi / 2}=36.74 \mu \mathrm{s}\right)$ on the ${ }^{14} \mathrm{~N}$ nuclear spin is much longer than the dephasing time $\left[T_{2}^{*}=8.1(2) \mu \mathrm{s}\right]$ of the electron spin qubit [63]. The decoherence effect on the electron spin during the ${ }^{14} \mathrm{~N}$ nuclear spin operation in the twoqubit gate experiments will dominate the fidelity decay. Hence 
TABLE I. Summary of the gate fidelity and duration for various QC schemes. DD-protected QC, dynamical-decoupling-protected quantum computing; SUPCODE QC, soft uniaxial positive control for orthogonal drift error quantum computing; GRAPE QC, gradient ascent pulse engineering quantum computing; AGQC, adiabatic geometric quantum computing.

\begin{tabular}{lccccc}
\hline \hline Scheme & DD-protected QC [62,63] & SUPCODE QC [60] & GRAPE QC [14] & AGQC [35] & B-NHQC \\
\hline Single-bit fidelity & 0.98 & $0.9961(2)$ & $0.999952(6)$ & $0.991(2)$ & $0.9922(4)$ \\
Two-bit fidelity & 0.90 & & $0.9920(1)$ & $0.94(2)$ & $0.965(4)$ \\
Single-bit gate duration $(\mu \mathrm{s})$ & 0.355 & 5.063 & 0.34 & 1 & 0.063 \\
Two-bit gate duration $(\mu \mathrm{s})$ & 50 & & 0.696 & 2 & 0.354 \\
\hline \hline
\end{tabular}

repeated applications of the $\mathrm{C}-\mathrm{Y}$ gate [65] on the electron spin and recording the dynamics of the quantum state constitute a better choice to estimate the gate fidelity as shown in Fig. 3(d). Figure 3(e) shows the fidelity of the final state to the ideal state for up to ten applications of the C-Y operation with no entanglement in the hybrid systems for an even number of gates. The result reveals $F_{s}(N)=A F_{g}^{N}+B$, where $A, B$, and two-qubit gate fidelity $F_{g}$ are fit parameters [65]. When $N$ is increased, the error of the $\mathrm{C}-\mathrm{Y}$ gate will accumulate, and state fidelity will decay. Here, we can obtain that the two-qubit gate fidelity is $F_{g}=0.965(4)$. The shortest possible time duration of the B-NHQC is advantageous to high fidelity due to the reduction of the ${ }^{14} \mathrm{~N}$ spin dephasing effect.

In Table I, we make a comparison between various quantum computation (QC) schemes with B-NHQC in experiment. The powers of the MW or radio-frequency drives are almost the same in these schemes. Since the B-NHQC scheme is the fastest geometric operation and the longest sequence duration is much less than the decoherence time, decoherence effects are expected to be negligible, and the fluctuations of control fields will be major errors. The systematic error in the Rabi frequency of the driving fields results in the leakage of the qubit's population into the noncomputational space. We notice that the duration of $32 \mathrm{~B}-\mathrm{NHQC}$ gates is about $1.8 \mu \mathrm{s}$, which is less than a SUPCODE gate [60]. However, our fidelity of 32 gates $(\sim 0.90)$ is smaller than the fidelity of a SUPCODE gate $(\sim 0.996)$. The reason is that the gate infidelity for fluctuation of the Rabi frequency is of the second order in $\delta \Omega / \Omega$, where $\delta \Omega$ denotes error in the Rabi frequency [57]. Fortunately, these control errors can be directly mitigated by combining multisegment operation of B-NHQC with composite-pulse architecture [50,60,66].

\section{QUANTUM ENHANCED METROLOGY WITH FULL GEOMETRIC GATES}

We further illustrate the advantage of QIP based on the full fast B-NHQC gates by implementing the entanglementenhanced parameter estimation algorithm [7-12] with the hybrid solid-state spin registers. By using independent sensors to estimate a parameter, we can achieve at best the standard quantum limit. However, it is believed that using entangled quantum sensor qubits, such as NOON states [7-12], one is able to achieve the fundamental HQL scaling.

As shown in Fig. 4(a), both the classical-classical (CC) and quantum-quantum (QQ) algorithms [8] are applied to probe the phase $\varphi$ [7-12], which can encode the target quantity to be measured. For the CC strategy, the interferometer signal is the expectation value $P=(1+\cos \varphi) / 2$, as shown in
Fig. 4(b) with the photon shot noise at the output denoted by error bars. Moreover, the electron and nuclear spin can be prepared in an entangled state with the QQ strategy. As shown in Fig. 4(a), we implement a phase gate on both sensor qubits, which brings the system to the two-qubit NOON state $\left(|00\rangle+e^{-2 \mathrm{i} \varphi}|11\rangle\right) / \sqrt{2}$. The interferometer signal is $P=$ $(1+\cos 2 \varphi) / 2$, as shown in Fig. 4(c) with the B-NHQC protocol. The quantum sensor is most sensitive to variations of the phase $(\Delta \varphi)$ at the point of the maximum slope, with the sensitivity limited by the uncertainty in the interferometer signal measurement $(\Delta P)$. The uncertainty of the hybrid spin register to small variations in phase, as depicted in the measurements, is given by $\Delta \varphi_{\min }=\sigma_{S}^{n} / d P$, where $\sigma_{S}^{n}$ is the standard deviation of the interferometer measurement after $n$ averages and $d P$ is the slope of the interferometer signal variation with phase [7-10]. By harnessing the entanglement of the electron and nuclear spins, the phase estimation uncertainties are reduced by 1.99(1) for the B-NHQC protocol compared with the $\mathrm{CC}$ algorithm, which is close to 2 to demonstrate the HQL. Moreover, to comprehensively and systematically analyze the experimental results, the duration times of $\mathrm{B}-\mathrm{NHQC}$ and
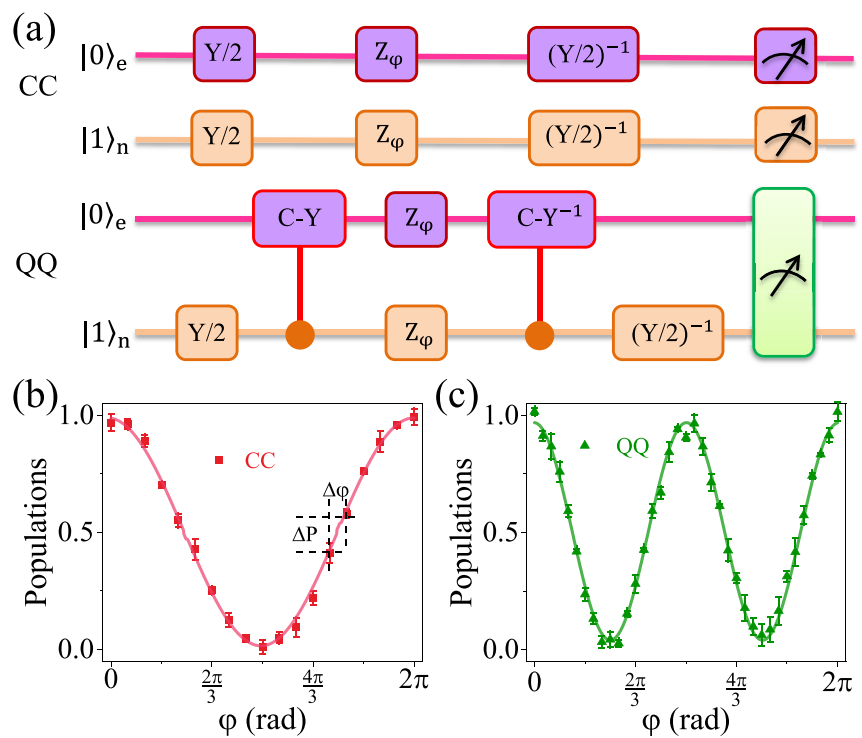

FIG. 4. Demonstration of the HQL with entanglement-enhanced phase estimation algorithm and B-NHQC gates. (a) The CC algorithm involves separable input states and separable measurements. The QQ algorithm involves general input states and general measurement schemes. (b) and (c) Phase relation of the two separable or entangled probes with B-NHQC gates. The phase gate $Z_{\varphi}$ is realized by adding a phase to the drive field for all operations. 
conventional NHQC protocols should be taken into consideration to discuss the measurement sensitivity $[41,43]$. The best measurement sensitivity is given by $S=\Delta \varphi_{\min } \sqrt{T_{t}}$, where $T_{t}$ is the total phase estimation algorithm time, which is dominated by the operation time of ${ }^{14} \mathrm{~N}$ nuclear spin. For B-NHQC, $T_{t}=85 \mu \mathrm{s}$ is less than that of the NHQC scheme $\left(T_{t}=90.5 \mu \mathrm{s}\right)$. Therefore the measurement sensitivity and bandwidth can be enhanced by mitigating the quantum decoherence effect and the instability of the experimental environment with short quantum state manipulation time.

\section{CONCLUSION}

In summary, fast and high-fidelity quantum coherent control is of fundamental significance in quantum computation, simulation, and metrology. We have implemented robust BNHQC-based single- and two-qubit gates with hybrid spin registers in diamond, which paves the way for full fast geometric QIP in practice. By using frequency-selective MW pulse coherent control technology [32,36], scalable geometric quantum computation can be implemented with a solid system [67]. Moreover, we further implement an entanglementenhanced phase estimation algorithm with an NV center with a fast and high-fidelity universal set of geometric gates. Beyond the conventional estimation paradigm, the best detection sensitivity and bandwidth are also improved in practice by benefiting from TOC quantum control. It will be further applied in high-resolution nuclear magnetic resonance detection at the nanoscale [68]. Beyond the demonstration in this paper, the B-NHQC protocol can be applied to other promising physical platforms, such as atoms, ions, and transmon or flux qubits, for fast and high-fidelity QIP.

\section{ACKNOWLEDGMENTS}

We thank Zheng-Yuan Xue for valuable discussions. This work is supported by the National Key Research and Development Program of China (Grant No. 2017YFA0304504), the National Natural Science Foundation of China (Grants No. 91850102 and No. 12005218), the Anhui Initiative in Quantum Information Technologies (Grant No. AHY130000), the Science Challenge Project (Grant No. TZ2018003), the Fundamental Research Funds for the Central Universities (Grant No. WK2030000020), and Excellent Youth Foundation of Hebei Scientific Committee (Grant No. F2019516002).

\section{APPENDIX A: NV CENTER IN DIAMOND}

In our experiment, the diamond sample is mounted at the focus of a home-built scanning confocal microscope, which is the same as the one described in Ref. [43]. A static magnetic field, $B_{0}=510 \mathrm{G}$, is applied along the NV symmetry axis ([111] crystal axis) and removes the degeneracy between the $\left|m_{S}=1\right\rangle$ and $\left|m_{S}=-1\right\rangle$ electron spin states. Under this magnetic field, the nitrogen nuclear spin can be optically polarized through excited level anticrossing effects, and the signal contrast can be enhanced up to $C=0.27$. After the optical pumping, $98 \%$ of the population occupies the $\left|m_{I}=1\right\rangle$ state of the nuclear spin. The NV centers studied in this paper are formed during chemical vapor deposition growth $[43,69]$.
The abundance of ${ }^{13} \mathrm{C}$ is at the natural level of $1.1 \%$. To beat the fluctuation of the photon counting, we repeat the experimental cycle at least $5 \times 10^{7}$ times.

\section{APPENDIX B: THE CONSTRUCTION OF THE NHQC GATE}

\section{Single-qubit gate}

In the single-qubit control case, experiments are implemented on the electron spin qubit while the nuclear spin is kept in state $\left|m_{I}=1\right\rangle$. When MW pulses with the frequencies $\omega_{1(2)}$ and initial phases $\phi_{1(2)}$ are applied, the total Hamiltonian of the electron spin qubit is

$$
\begin{aligned}
H_{s}=D S_{z}^{2}+\gamma_{e} B S_{z}+ & {\left[\gamma_{e} B_{1} \cos \left(\omega_{1} t+\phi_{1}\right)\right.} \\
& \left.+\gamma_{e} B_{2} \cos \left(\omega_{2} t+\phi_{2}\right)\right] S_{x} .
\end{aligned}
$$

We apply the unitary transformation

$$
V=\left(\begin{array}{ccc}
e^{-i \omega_{1} t} & 0 & 0 \\
0 & 1 & 0 \\
0 & 0 & e^{-i \omega_{2} t}
\end{array}\right)
$$

to generate the transformed Hamiltonian $H_{r}=V^{\dagger} H V+$ $i\left(\partial V^{\dagger} / \partial t\right) V$, which is analogous to transforming to the rotating frame in a spin- $1 / 2$ system. The result is

$$
H_{r}=\left(\begin{array}{ccc}
D+\gamma_{e} B-\omega_{2} & \frac{\Omega_{2}}{2} e^{-i \phi_{2}} & 0 \\
\frac{\Omega_{2}}{2} e^{i \phi_{2}} & 0 & \frac{\Omega_{1}}{2} e^{i \phi_{1}} \\
0 & \frac{\Omega_{1}}{2} e^{-i \phi_{1}} & D-\gamma_{e} B-\omega_{1}
\end{array}\right),
$$

where $\Omega_{1}=\frac{\sqrt{2}}{2} \gamma_{e} B_{1}$ and $\Omega_{2}=\frac{\sqrt{2}}{2} \gamma_{e} B_{2}$. By setting $\omega_{1}=D+$ $\gamma_{e} B$ and $\omega_{2}=D-\gamma_{e} B$, we can get

$$
\begin{aligned}
H & =\left[\frac{\Omega_{1}(t)}{2} e^{i \phi_{1}}|0\rangle+\frac{\Omega_{2}(t)}{2} e^{i \phi_{2}}|1\rangle\right]\langle a|+\text { H.c. } \\
& =\frac{\Omega(t)}{2} e^{i \phi_{2}}|b\rangle\langle a|+\text { H.c., }
\end{aligned}
$$

where $|b\rangle=e^{i \phi} \sin \frac{\theta}{2}|0\rangle+\cos \frac{\theta}{2}|1\rangle$ is a bright state with $\tan \frac{\theta}{2}=\frac{\Omega_{1}}{\Omega_{2}}, \phi=\phi_{1}-\phi_{2}$, and $\Omega(t)=\sqrt{\Omega_{1}^{2}(t)+\Omega_{2}^{2}(t)}$. As shown in Eq. (1), the bright state $|b\rangle$ interacts with the state $|a\rangle$, while it decouples from the dark state $|d\rangle=\cos \frac{\theta}{2}|0\rangle-e^{-i \phi} \sin \frac{\theta}{2}|1\rangle$.

The NHQC scheme $[19,36]$ can be realized with a singleloop scenario by engineering the quantum system to evolve along with the red slice-shaped path in the Bloch sphere shown in Fig. 1(a). In the first segment [0, $\tau / 2]$, we set $\phi_{2}=0$, and then $H$ is reduced to $H_{a}=\frac{\Omega}{2}(|b\rangle\langle a|+| a\rangle\langle b|)$. The corresponding evolution operator is $U_{a}=|d\rangle\langle d|-$ $i(|b\rangle\langle a|+| a\rangle\langle b|)$. In the second segment $[\tau / 2, \tau]$, we change the MW phase $\phi_{2}$ to $\tilde{\phi}_{2}=\pi+\gamma$. Then $H$ is reduced to $H_{b}=-\frac{\Omega}{2}\left(e^{i \gamma}|b\rangle\left\langle a\left|+e^{-i \gamma}\right| a\right\rangle\langle b|\right)$, and the corresponding evolution operator is $U_{b}=|d\rangle\langle d|+i\left(e^{i \gamma}|b\rangle\left\langle a\left|+e^{-i \gamma}\right| a\right\rangle\langle b|\right)$. In this way, in the qubit computational basis $\{|0\rangle,|1\rangle\}$, the induced gate operation will be

$$
U_{G}(\gamma, \theta, \phi)=U_{b} U_{a}=e^{i(\gamma / 2)} e^{-i(\gamma / 2) \vec{n} \cdot \vec{\sigma}},
$$

which describes a rotation around the $\vec{n}=(\sin \theta \cos \phi$, $\sin \theta \sin \phi, \cos \theta)$ axis by a $\gamma$ angle. In the conventional NHQC construction, the cyclic path evolution area is set as 


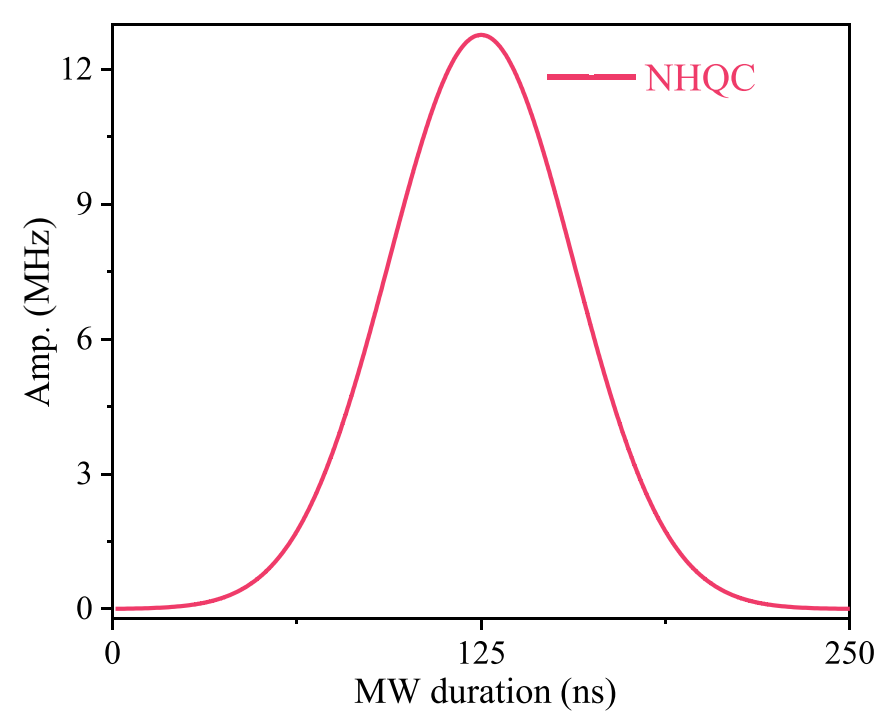

FIG. 5. The wave form of the $4 \sigma$ truncated Gaussian shape for the conventional NHQC gate. The maximum of the Rabi frequency with the Gaussian $4 \sigma$ truncated pulse is $12.76 \mathrm{MHz}$ for electron spin operation of the NV center.

$\int_{0}^{\tau} \Omega(t) d t=2 \pi . \tau$ is the entire evolution time, which is separated into two equal segments. The geometric control of the ${ }^{14} \mathrm{~N}$ nuclear spin is the same with this single-qubit gate due to similar energy levels.

In our geometric shortcut circular path of the BNHQC scheme, the Hamiltonian equation (1) is equivalent

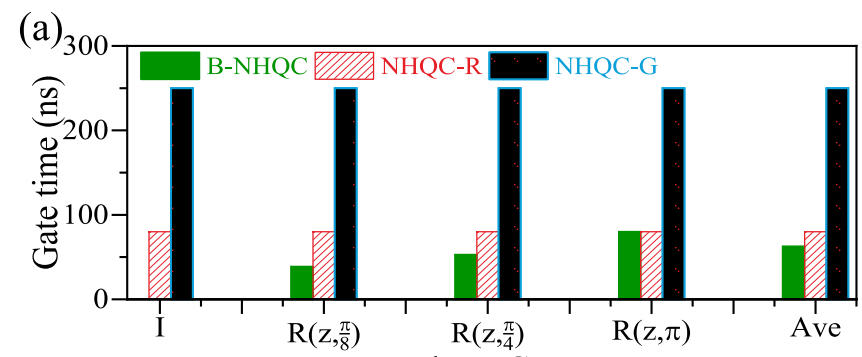

(b)

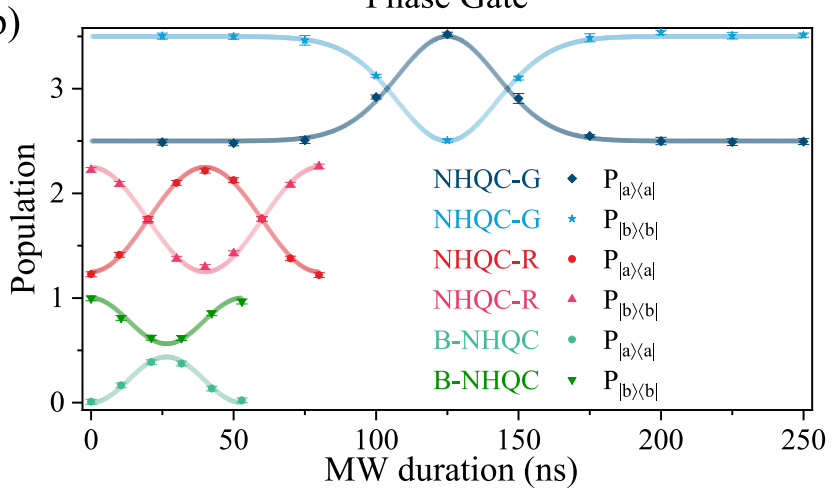

FIG. 6. (a) Comparison of the experimental gate time for $\theta=$ $\pi / 8, \pi / 4$, and $\pi$. NHQC-G (NHGC-R) denotes the NHQC gate with a Gaussian (rectangular) wave-form modulation. B-NHQC denotes the B-NHQC gate with a rectangular wave-form modulation. (b) State evolutions during $T=R\left(\hat{z}, \frac{\pi}{4}\right)$ with $\mathrm{B}-\mathrm{NHQC}$ and the conventional NHQC paths.

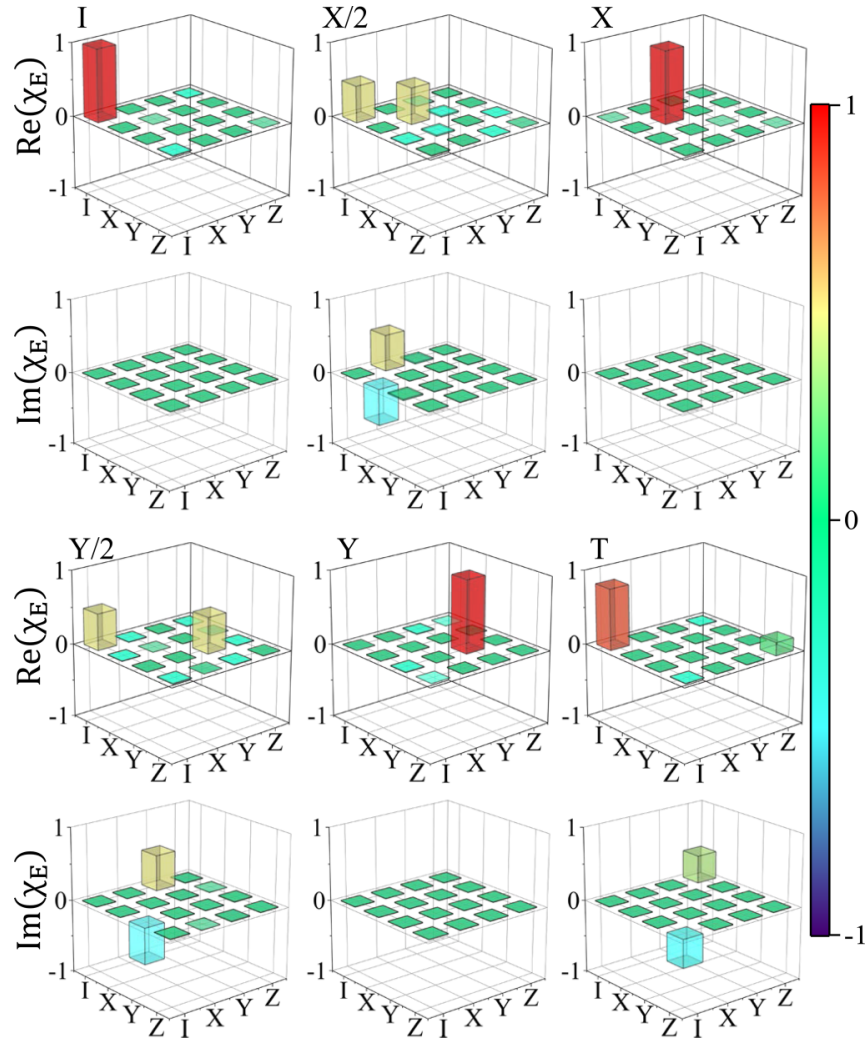

FIG. 7. QPT of B-NHQC gates. A set of the rotations $\{I, X / 2, X, Y / 2, Y, T\}$ are used to generate input states and analyze the processes. The bar charts of the real and imaginary parts of $\chi_{E}$ for specific gates $\{I, X / 2, X, Y / 2, Y, T\}$ give an average process fidelity of $F_{a v}=0.984(2)$. The labels on the $X$ and $Y$ axes correspond to the operators in the basis set $\left\{I, \sigma_{x}, \sigma_{y}, \sigma_{z}\right\}$ of the $|0\rangle,|1\rangle$ subspace.

to $H_{1}=\Delta|a\rangle\langle a|+\Omega\left(e^{i \phi}|b\rangle\langle a|+\right.$ H.c. $)$, where $\Delta=-2(\gamma-$ $\pi) / \tau$ and $\phi$ is time dependent. The dark state $|d\rangle$ is decoupled from the dynamics. The bright state $|b\rangle$ undergoes excitation to ancillary state $|a\rangle$. In our case, $\Omega(t)$ is a rectangular pulse, and $H_{1}$ is time independent during the dynamics. Thus the expected value of the energy is conserved, remaining zero during the evolution for any initial state in the subspace spanned by $\left|m_{s}= \pm 1\right\rangle$ and ensuring the absence of a dynamic phase.

In 2013, the NHQC single-qubit gates were implemented in the transmon system [27]. Since then, for realistic quantum control of the physical system, the Gaussian $4 \sigma$ truncated wave form $[27,29,32,70]$ is preferred in the experimental demonstration, as shown in Fig. 5. Here, we can take the Gaussian wave-form modulation control pulses to avoid leakage [71]. We can see that NHQC with a Gaussian wave-form modulation takes a long time, which is more than three times longer than for the B-NHQC case on average as shown in Fig. 6(a). However, the global dynamical control process of the geometric gate for NHQC, which is quite different with $\mathrm{B}-\mathrm{NHQC}$, is independent of the shape of modulation as shown in Fig. 6(b).

\section{Two-qubit gate}

In the construction of two-qubit gate, we use the electron spin state as the target state and the nonzero host nitrogen 
nuclear spin $\left(I=1\right.$ for $\left.{ }^{14} \mathrm{~N}\right)$ of the NV center as the control qubit. The Hamiltonian can be expressed as

$$
H_{s}=D S_{z}^{2}+\gamma_{e} B S_{z}+P I_{z}^{2}+\gamma_{n} B I_{z}+A_{z z} S_{z} I_{z} .
$$

Here, $S_{z}$ and $I_{z}$ are the $z$ components of the spin-1 operators for the electron and nitrogen nuclear spins $\left({ }^{14} \mathrm{~N}\right)$, respectively. $\gamma_{e}\left(\gamma_{n}\right)$ is the electronic $\left({ }^{14} \mathrm{~N}\right.$ nuclear) gyromagnetic ratio. The zero-field splitting $D=2.87 \mathrm{GHz}$, the nuclear quadrupolar splitting $P=-4.95 \mathrm{MHz}$, and the hyperfine interaction between the $\mathrm{NV}$ electron and the ${ }^{14} \mathrm{~N}$ nuclear spin $A=2.16 \mathrm{MHz}$. By applying state-selective MW and radiofrequency (rf) pulses, we can couple different energy levels. For arbitrary sublevels of the electron spin ground state, the
${ }^{14} \mathrm{~N}$ nuclear spin system has a lambda $(\Lambda)$ energy structure, which is preferable to realize the B-NHQC scheme $[24,40]$.

\section{APPENDIX C: QPT OF THE SINGLE-QUBIT GATE}

We characterize the single-qubit B-NHQC gates through the QPT method [43]. The experimental process matrices $\chi_{E}$ of six specific geometric gates $\{I, X / 2, X, Y / 2, Y, T\}$ are shown in Fig. 7, with the fidelities of $0.988(4), 0.981(3)$, $0.982(3), 0.981(6), 0.987(7), 0.983(4)$, respectively. Here, the quantum process fidelity is calculated with $F=\left|\operatorname{Tr}\left(\chi_{E} \chi_{i d}^{\dagger}\right)\right|$ by comparing with the idea operation $\chi_{i d}$. The major contribution to the QPT infidelity comes from state preparation and detection errors $[32,33,35,59]$.
[1] F. Arute, K. Arya, R. Babbush, D. Bacon, J. C. Bardin, R. Barends, R. Biswas, S. Boixo, F. G. Brandao, D. A. Buell, B. Burkett, Y. Chen, Z. Chen, B. Chiaro, R. Collins, W. Courtney, A. Dunsworth, E. Farhi, B. Foxen, A. Fowler et al., Quantum supremacy using a programmable superconducting processor, Nature (London) 574, 505 (2019).

[2] Y. Alexeev, D. Bacon, K. R. Brown, R. Calderbank, L. D. Carr, F. T. Chong, B. DeMarco, D. Englund, E. Farhi, B. Fefferman, A. V. Gorshkov, A. Houck, J. Kim, S. Kimmel, M. Lange, S. Lloyd, M. D. Lukin, D. Maslov, P. Maunz, C. Monroe, J. Preskill, M. Roetteler, M. J. Savage, and J. Thompson, Quantum computer systems for scientific discovery, PRX Quantum 2, 017001 (2021).

[3] D. S. Abrams and S. Lloyd, Quantum Algorithm Providing Exponential Speed Increase for Finding Eigenvalues and Eigenvectors, Phys. Rev. Lett. 83, 5162 (1999).

[4] A. W. Harrow, A. Hassidim, and S. Lloyd, Quantum Algorithm for Linear Systems of Equations, Phys. Rev. Lett. 103, 150502 (2009).

[5] I. Buluta and F. Nori, Quantum simulators, Science 326, 108 (2009).

[6] I. M. Georgescu, S. Ashhab, and F. Nori, Quantum simulation, Rev. Mod. Phys. 86, 153 (2014).

[7] V. Giovannetti, S. Lloyd, and L. Maccone, Quantum-enhanced measurements: Beating the standard quantum limit, Science 306, 1330 (2004)

[8] V. Giovannetti, S. Lloyd, and L. Maccone, Quantum Metrology, Phys. Rev. Lett. 96, 010401 (2006).

[9] S. Pirandola, B. R. Bardhan, T. Gehring, C. Weedbrook, and S. Lloyd, Advances in photonic quantum sensing, Nat. Photon. 12, 724 (2018).

[10] L. Pezze, A. Smerzi, M. K. Oberthaler, R. Schmied, and P. Treutlein, Quantum metrology with nonclassical states of atomic ensembles, Rev. Mod. Phys. 90, 035005 (2018).

[11] Y. Dong, X.-D. Chen, G.-C. Guo, and F.-W. Sun, Reviving the precision of multiple entangled probes in an open system by simple $\pi$-pulse sequences, Phys. Rev. A 94, 052322 (2016).

[12] G.-Q. Liu, Y.-R. Zhang, Y.-C. Chang, J.-D. Yue, H. Fan, and X.-Y. Pan, Demonstration of entanglement-enhanced phase estimation in solid, Nat. Commun. 6, 6726 (2015).

[13] F. Xu, X. Ma, Q. Zhang, H.-K. Lo, and J.-W. Pan, Secure quantum key distribution with realistic devices, Rev. Mod. Phys. 92, 025002 (2020).
[14] X. Rong, J. Geng, F. Shi, Y. Liu, K. Xu, W. Ma, F. Kong, Z. Jiang, Y. Wu, and J. Du, Experimental fault-tolerant universal quantum gates with solid-state spins under ambient conditions, Nat. Commun. 6, 8748 (2015).

[15] Y. Dong, Y. Zheng, S. Li, C.-C. Li, X.-D. Chen, G.-C. Guo, and F.-W. Sun, Non-Markovianity-assisted high-fidelity DeutschJozsa algorithm in diamond, npj Quantum Inf. 4, 3 (2018).

[16] Y. Dong, X.-D. Chen, G.-C. Guo, and F.-W. Sun, Robust scalable architecture for a hybrid spin-mechanical quantum entanglement system, Phys. Rev. B 100, 214103 (2019).

[17] A. Bourassa, C. P. Anderson, K. C. Miao, M. Onizhuk, H. Ma, A. L. Crook, H. Abe, J. Ul-Hassan, T. Ohshima, N. T. Son, G. Galli, and D. D. Awschalom, Entanglement and control of single nuclear spins in isotopically engineered silicon carbide, Nat. Mater. 19, 1319 (2020).

[18] D. Yuan, Y. Guan, W. Chen, H. Zhao, S. Yu, C. Luo, Y. Tan, T. Xie, X. Wang, Z. Sun, D. H. Zhang, and X. Yang, Observation of the geometric phase effect in the $\mathrm{H}+\mathrm{HD} \rightarrow \mathrm{H}_{2}+\mathrm{D}$ reaction, Science 362, 1289 (2018).

[19] P. Zanardi and M. Rasetti, Holonomic quantum computation, Phys. Lett. A 264, 94 (1999).

[20] L.-M. Duan, J. I. Cirac, and P. Zoller, Geometric manipulation of trapped ions for quantum computation, Science 292, 1695 (2001).

[21] K. Arai, J. Lee, C. Belthangady, D. R. Glenn, H. Zhang, and R. L. Walsworth, Geometric phase magnetometry using a solidstate spin, Nat. Commun. 9, 4996 (2018).

[22] E. Sjöqvist, D.-M. Tong, L. M. Andersson, B. Hessmo, M Johansson, and K. Singh, Non-adiabatic holonomic quantum computation, New J. Phys. 14, 103035 (2012).

[23] G. F. Xu, J. Zhang, D. M. Tong, E. Sjöqvist, and L. C. Kwek, Nonadiabatic Holonomic Quantum Computation in Decoherence-Free Subspaces, Phys. Rev. Lett. 109, 170501 (2012).

[24] B.-J. Liu, X.-K. Song, Z.-Y. Xue, X. Wang, and M.-H. Yung, Plug-and-Play Approach to Nonadiabatic Geometric Quantum Gates, Phys. Rev. Lett. 123, 100501 (2019).

[25] M. Johansson, E. Sjöqvist, L. M. Andersson, M. Ericsson, B. Hessmo, K. Singh, and D. M. Tong, Robustness of nonadiabatic holonomic gates, Phys. Rev. A 86, 062322 (2012).

[26] E. Herterich and E. Sjöqvist, Single-loop multiple-pulse nonadiabatic holonomic quantum gates, Phys. Rev. A 94, 052310 (2016). 
[27] A. A. Abdumalikov Jr, J. M. Fink, K. Juliusson, M. Pechal, S. Berger, A. Wallraff, and S. Filipp, Experimental realization of non-Abelian non-adiabatic geometric gates, Nature (London) 496, 482 (2013).

[28] T. Yan, B.-J. Liu, K. Xu, C. Song, S. Liu, Z. Zhang, H. Deng, Z. Yan, H. Rong, K. Huang, M. H. Yung, Y. Chen, and D. Yu, Experimental Realization of Nonadiabatic Shortcut to Non-Abelian Geometric Gates, Phys. Rev. Lett. 122, 080501 (2019).

[29] Y. Xu, Z. Hua, T. Chen, X. Pan, X. Li, J. Han, W. Cai, Y. Ma, H. Wang, Y. P. Song, Z. Y. Xue, and L. Sun, Experimental Implementation of Universal Nonadiabatic Geometric Quantum Gates in a Superconducting Circuit, Phys. Rev. Lett. 124, 230503 (2020).

[30] G. Feng, G. Xu, and G. Long, Experimental Realization of Nonadiabatic Holonomic Quantum Computation, Phys. Rev. Lett. 110, 190501 (2013).

[31] Z. Zhu, T. Chen, X. Yang, J. Bian, Z.-Y. Xue, and X. Peng, Single-Loop and Composite-Loop Realization of Nonadiabatic Holonomic Quantum Gates in a Decoherence-Free Subspace, Phys. Rev. Appl. 12, 024024 (2019).

[32] C. Zu, W.-B. Wang, L. He, W.-G. Zhang, C.-Y. Dai, F. Wang, and L.-M. Duan, Experimental realization of universal geometric quantum gates with solid-state spins, Nature (London) $\mathbf{5 1 4}$, 72 (2014).

[33] S. Arroyo-Camejo, A. Lazariev, S. W. Hell, and G. Balasubramanian, Room temperature high-fidelity holonomic single-qubit gate on a solid-state spin, Nat. Commun. 5, 4870 (2014).

[34] K. Nagata, K. Kuramitani, Y. Sekiguchi, and H. Kosaka, Universal holonomic quantum gates over geometric spin qubits with polarised microwaves, Nat. Commun. 9, 3227 (2018).

[35] Y.-Y. Huang, Y.-K. Wu, F. Wang, P.-Y. Hou, W.-B. Wang, W.-G. Zhang, W.-Q. Lian, Y.-Q. Liu, H.-Y. Wang, H.-Y. Zhang, L. He, X. Y. Chang, Y. Xu, and L. M. Duan, Experimental Realization of Robust Geometric Quantum Gates with Solid-State Spins, Phys. Rev. Lett. 122, 010503 (2019).

[36] Y. Dong, S.-C. Zhang, Y. Zheng, H.-B. Lin, L.-K. Shan, X.D. Chen, W. Zhu, G.-Z. Wang, G.-C. Guo, and F.-W. Sun, Experimental Implementation of Universal Holonomic Quantum Computation on Solid-State Spins with Optimal Control, Phys. Rev. Appl. 16, 024060 (2021).

[37] N. V. Vitanov, A. A. Rangelov, B. W. Shore, and K. Bergmann, Stimulated Raman adiabatic passage in physics, chemistry, and beyond, Rev. Mod. Phys. 89, 015006 (2017).

[38] D. Guéry-Odelin, A. Ruschhaupt, A. Kiely, E. Torrontegui, S. Martínez-Garaot, and J. G. Muga, Shortcuts to adiabaticity: Concepts, methods, and applications, Rev. Mod. Phys. 91, 045001 (2019).

[39] S.-B. Zheng, C.-P. Yang, and F. Nori, Comparison of the sensitivity to systematic errors between nonadiabatic non-Abelian geometric gates and their dynamical counterparts, Phys. Rev. A 93, 032313 (2016).

[40] S. Li, T. Chen, and Z.-Y. Xue, Fast holonomic quantum computation on superconducting circuits with optimal control, Adv. Quantum Technol. 3, 2000001 (2020).

[41] J. F. Barry, J. M. Schloss, E. Bauch, M. J. Turner, C. A. Hart, L. M. Pham, and R. L. Walsworth, Sensitivity optimization for NV-diamond magnetometry, Rev. Mod. Phys. 92, 015004 (2020).
[42] Y. Dong, J.-Y. Xu, S.-C. Zhang, Y. Zheng, X.-D. Chen, W. Zhu, G.-Z. Wang, G.-C. Guo, and F.-W. Sun, Compositepulse enhanced room-temperature diamond magnetometry, Funct. Diamond 1, 125 (2021).

[43] Y. Dong, S.-C. Zhang, H.-B. Lin, X.-D. Chen, W. Zhu, G.-Z Wang, G.-C. Guo, and F.-W. Sun, Quantifying the performance of multipulse quantum sensing, Phys. Rev. B 103, 104104 (2021).

[44] Y. Sekiguchi, N. Niikura, R. Kuroiwa, H. Kano, and H. Kosaka, Optical holonomic single quantum gates with a geometric spin under a zero field, Nat. Photon. 11, 309 (2017).

[45] B. B. Zhou, P. C. Jerger, V. O. Shkolnikov, F. J. Heremans, G. Burkard, and D. D. Awschalom, Holonomic Quantum Control by Coherent Optical Excitation in Diamond, Phys. Rev. Lett. 119, 140503 (2017).

[46] A. T. Rezakhani, W.-J. Kuo, A. Hamma, D. A. Lidar, and P. Zanardi, Quantum Adiabatic Brachistochrone, Phys. Rev. Lett. 103, 080502 (2009).

[47] X. Chen, I. Lizuain, A. Ruschhaupt, D. Guéry-Odelin, and J. G. Muga, Shortcut to Adiabatic Passage in Two-and Three-Level Atoms, Phys. Rev. Lett. 105, 123003 (2010).

[48] Y. Ban, X. Chen, E. Y. Sherman, and J. G. Muga, Fast and Robust Spin Manipulation in a Quantum Dot by Electric Fields, Phys. Rev. Lett. 109, 206602 (2012).

[49] D. Daems, A. Ruschhaupt, D. Sugny, and S. Guerin, Robust Quantum Control by a Single-Shot Shaped Pulse, Phys. Rev. Lett. 111, 050404 (2013).

[50] G. F. Xu, D. M. Tong, and E. Sjöqvist, Path-shortening realizations of nonadiabatic holonomic gates, Phys. Rev. A 98, 052315 (2018).

[51] X. Wang, M. Allegra, K. Jacobs, S. Lloyd, C. Lupo, and M. Mohseni, Quantum Brachistochrone Curves as Geodesics: Obtaining Accurate Minimum-Time Protocols for the Control of Quantum Systems, Phys. Rev. Lett. 114, 170501 (2015).

[52] A. Carlini, A. Hosoya, T. Koike, and Y. Okudaira, TimeOptimal Quantum Evolution, Phys. Rev. Lett. 96, 060503 (2006).

[53] A. Carlini and T. Koike, Time-optimal transfer of coherence, Phys. Rev. A 86, 054302 (2012).

[54] J. Geng, Y. Wu, X. Wang, K. Xu, F. Shi, Y. Xie, X. Rong, and J. Du, Experimental Time-Optimal Universal Control of Spin Qubits in Solids, Phys. Rev. Lett. 117, 170501 (2016).

[55] S. Lloyd, Ultimate physical limits to computation, Nature (London) 406, 1047 (2000)

[56] M. R. Lam, N. Peter, T. Groh, W. Alt, C. Robens, D. Meschede, A. Negretti, S. Montangero, T. Calarco, and A. Alberti, Demonstration of Quantum Brachistochrones between Distant States of an Atom, Phys. Rev. X 11, 011035 (2021).

[57] B.-J. Liu, Z.-Y. Xue, and M.-H. Yung, Brachistochronic nonadiabatic holonomic quantum control, arXiv:2001.05182.

[58] T. Chen, P. Shen, and Z.-Y. Xue, Robust and Fast Holonomic Quantum Gates with Encoding on Superconducting Circuits, Phys. Rev. Appl. 14, 034038 (2020).

[59] F. Kleißler, A. Lazariev, and S. Arroyo-Camejo, Universal, high-fidelity quantum gates based on superadiabatic, geometric phases on a solid-state spin-qubit at room temperature, npj Quantum Inf. 4, 49 (2018).

[60] X. Rong, J. Geng, Z. Wang, Q. Zhang, C. Ju, F. Shi, C.-K. Duan, and J. Du, Implementation of Dynamically Corrected Gates on a 
Single Electron Spin in Diamond, Phys. Rev. Lett. 112, 050503 (2014).

[61] F. Jelezko, T. Gaebel, I. Popa, M. Domhan, A. Gruber, and J. Wrachtrup, Observation of Coherent Oscillation of a Single Nuclear Spin and Realization of a Two-Qubit Conditional Quantum Gate, Phys. Rev. Lett. 93, 130501 (2004).

[62] J. Zhang, A. M. Souza, F. D. Brandao, and D. Suter, Protected Quantum Computing: Interleaving Gate Operations with Dynamical Decoupling Sequences, Phys. Rev. Lett. 112, 050502 (2014).

[63] J. Zhang and D. Suter, Experimental Protection of TwoQubit Quantum Gates against Environmental Noise by Dynamical Decoupling, Phys. Rev. Lett. 115, 110502 (2015).

[64] D. A. Golter, T. K. Baldwin, and H. Wang, Protecting a Solid-State Spin from Decoherence using Dressed Spin States, Phys. Rev. Lett. 113, 237601 (2014).

[65] J. M. Chow, J. M. Gambetta, A. D. Corcoles, S. T. Merkel, J. A. Smolin, C. Rigetti, S. Poletto, G. A. Keefe, M. B. Rothwell, J. R. Rozen, M. B. Ketchen, and M. Steffen, Universal Quantum Gate Set Approaching Fault-Tolerant Thresholds with Superconducting Qubits, Phys. Rev. Lett. 109, 060501 (2012).
[66] G. T. Genov, D. Schraft, N. V. Vitanov, and T. Halfmann, Arbitrarily Accurate Pulse Sequences for Robust Dynamical Decoupling, Phys. Rev. Lett. 118, 133202 (2017).

[67] C. E. Bradley, J. Randall, M. H. Abobeih, R. C. Berrevoets, M. J. Degen, M. A. Bakker, M. Markham, D. J. Twitchen, and T. H. Taminiau, A Ten-Qubit Solid-State Spin Register with Quantum Memory up to One Minute, Phys. Rev. X 9, 031045 (2019).

[68] N. Aslam, M. Pfender, P. Neumann, R. Reuter, A. Zappe, F. F. de Oliveira, A. Denisenko, H. Sumiya, S. Onoda, J. Isoya, and J. Wrachtrup, Nanoscale nuclear magnetic resonance with chemical resolution, Science 357, 67 (2017).

[69] B. Zhao, Y. Dong, S. Zhang, X. Chen, W. Zhu, and F. Sun, Improving the NV generation efficiency by electron irradiation, Chin. Opt. Lett. 18, 080201 (2020).

[70] Y. Xu, W. Cai, Y. Ma, X. Mu, L. Hu, T. Chen, H. Wang, Y. P. Song, Z.-Y. Xue, Z. Q. Yin, and L. Sun, Single-Loop Realization of Arbitrary Nonadiabatic Holonomic Single-Qubit Quantum Gates in a Superconducting Circuit, Phys. Rev. Lett. 121, 110501 (2018).

[71] F. Motzoi, J. M. Gambetta, P. Rebentrost, and F. K. Wilhelm, Simple Pulses for Elimination of Leakage in Weakly Nonlinear Qubits, Phys. Rev. Lett. 103, 110501 (2009). 